MATEC Web of Conferences 19, 01017 (2014)

DOI: $10.1051 /$ matecconf/ 20141901017

(C) Owned by the authors, published by EDP Sciences, 2014

\title{
Energy and resource saving raw materials for dactyloscopy
}

\author{
Lyubov F. Ikonnikova ${ }^{1}$, KseniyaV. Ikonnikova and Ekaterina A. Koltunova
}

National Research Tomsk Polytechnic University, 634050 Tomsk, Russia

\begin{abstract}
The "developing" properties of ferromagnetic fingerprint powders are defined by the chemical nature and the particle sizes of the pigment which form acidity of its surface, adhesive and magnetic properties, and color spectrum. The optimum ratio of these physical and chemical parameters is observed in the pigment consisting of iron-containing sludge tempered at $800^{\circ} \mathrm{C}$, which contains $\alpha-\mathrm{Fe}_{2} \mathrm{O}_{3}$ and impurity of clay materials.
\end{abstract}

\section{Introduction}

The problem concerning the application of iron-containing sludge for ground water purification has not been solved yet. Deironing at Tomsk water intake is carried out by means of simplified water aeration. More than 600 tons of iron-containing sludge per year are piled up after precipitation and filtration. The products extracted from the sludge suitable for the application in national economy are not produced and the fallouts (sludging) are buried. However, the sludge tempered at different temperatures produces fine-dispersed iron oxide powders of different colors, which physical and chemical parameters are not worse than the same parameters of the pigments obtained from the other raw materials (natural ore or iron-containing reagents). The flow chart of obtaining pigment using these methods is multistage, energy and resource costly [1]. The production of the pigment from water purification sludge is more energy-profitable and recourse-saving which emphasizes the ecological and economic significance of the product obtained by the technogenic processing of raw materials. Taking all above mentioned into account, the problem concerning the investigation and finding the areas of application of the iron oxide pigments from water treatment sludge is urgent. Polychromy and fine dispersion of the pigments attracts attention of building industry, the profitability of this pigment application for preparation of cement-sand colored compositions and painting materials intended for different purposes is also presented in the given article [2]. There is no development available concerning the application of the magnetic properties of the pigments from iron-containing sludge.

The aim of this work is to study the possibility of the production of fingerprint ferromagnetic powders from wastewater treatment sludge.

The stated objective meets the requirements of the domestic fingerprint analysis concerning providing this branch by ferromagnetic powders with high affinity to sweat - fatty substance and satisfactory fingerprint informativeness.

Ferromagnetic powders are applied with the use of a magnetic applicator and consist either of a partially oxidized metallic iron (to reduce its pyrophoric), or a mixture of iron and iron oxide pigment, which play a broad spectrum of roles:

- metallic iron forms "pile" of the magnetic brush and is a carrier of the pigment;

- iron-oxide pigments (of a wide spectrum of colors and tints) pollinate and make visually distinguishable colorless sweat-fatty fingerprint on the track-carrying surface. Dactyloscopic informativeness of the obtained print is determined by optimum relationship of magnetic and adhesive properties of the dye (pigment) - powder (dosed pollination and pigment adhesion to the print.

\section{Experimental part}

The subjects of inquiry in the given paper were as follows:

- regular fingerprint powder used by the field criminalists as a universal one (sample №1);

- metal iron obtained by means of the method of electric conductor explosion (sample No. 2);

- the pigment of iron-containing sludge tempered at $800^{\circ} \mathrm{C}$ (sample №3);

${ }^{1}$ Corresponding author: ikonna@tpu.ru

This is an Open Access article distributed under the terms of the Creative Commons Attribution License 4.0, which permits unrestricted use, distribution, and reproduction in any medium, provided the original work is properly cited. 
- simulated fingerprint powder consisting of the pigment mixture (compound) from metallic iron and iron-containing sludge (sample №4).

Technological production criteria allow some error in the composition, which leads to the difference in the morphology and properties of the various consignment of goods not only by different manufacturers, but by the single producer as well.

Therefore, the purpose of this study was to determine the chemical composition and structure of the samples, the shape and size of the particles, the surface activity of the samples concerning pure water (based on sweat fatty secretion), the adhesive ability to fat (of a stable part of sweat fatty secretion). The specified parameters were determined by the methods of X-ray phase analysis, infra red spectroscopy, electron microscopy, and pH-metry.

\section{Results and discussion}

The results showed that all samples are hydrophilic nano-sized particles of spheroidal shape, the surface acidity of the particles is close to neutral one (Figure 1, Table 1). It provides the compatibility and good adhesion of powders to sweat fatty print (as well as its neutral nature of powder).

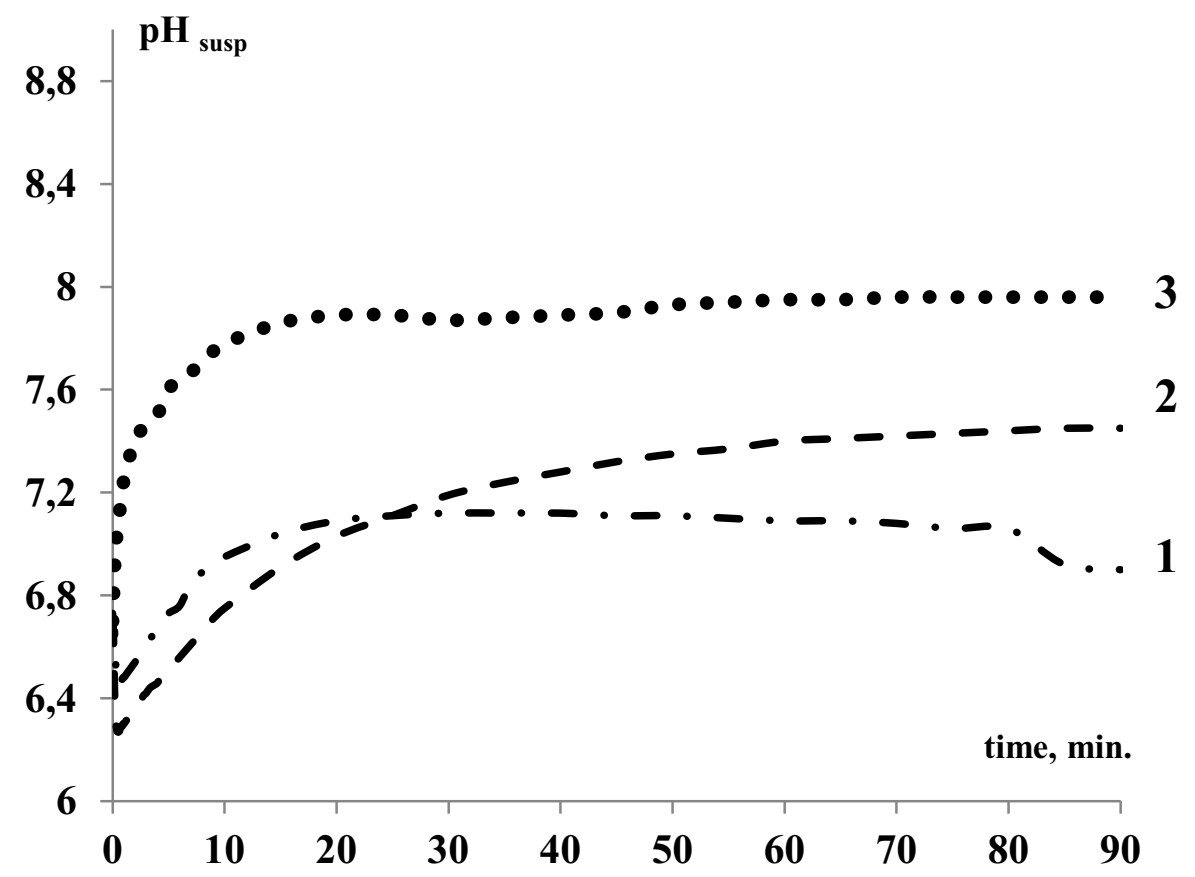

Figure 1. The variations in the acidity of an aqueous suspension within certain time period: 1 - regular fingerprint powder, 2 - pigment consisting of iron-containing sludge, 3 - metallic iron (obtained by means of electric conductor explosion).

The composition of all samples included the most thermodynamically stable configuration of $\alpha$ $\mathrm{Fe}_{2} \mathrm{O}_{3}$ in different conditions: in fingerprint powder and metallic iron, samples №1 and №2, in the configuration of encapsulating iron particle of the protective film possessing thickness of 1-2 nm, and in the pigment of iron-containing sludge, sample №3, in a massive state. The magnetic susceptibility of $\alpha-\mathrm{Fe}_{2} \mathrm{O}_{3}$ (according to the literature data) has much lower values than those of metallic iron, which is crucial for dosed pigment capture. 
The $2^{\text {nd }}$ International Youth Forum "Smart Grids"

Table 1. Comparative characteristics of the test specimen

\begin{tabular}{|c|c|c|c|c|c|}
\hline \multirow{2}{*}{$№$} & \multicolumn{2}{|c|}{ Particle sizes, $\mathrm{mkm}$} & \multicolumn{2}{c|}{$\mathrm{pH}_{\text {susp }}$ time } \\
\cline { 3 - 5 } & Specimen characteristic & micro & macro & $10^{\prime \prime}$ & $90^{\prime}$ \\
\hline 1 & $\begin{array}{c}\text { fingerprint powder } \\
\mathrm{Fe}+\alpha-\mathrm{Fe}_{2} \mathrm{O}_{3}(10 \%)\end{array}$ & 0,200 & $4 \div 30$ & 6,4 & 6,7 \\
\hline 2 & $\begin{array}{c}\text { metallic iron } \\
\mathrm{Fe}+\alpha-\mathrm{Fe}_{2} \mathrm{O}_{3}(20,6 \%)\end{array}$ & $0,1 \div 0,15$ & $1 \div 3$ & 6,3 & 7,4 \\
\hline 3 & $\begin{array}{c}\text { pigment of iron-containing sludge } \\
\alpha-\mathrm{Fe}_{2} \mathrm{O}_{3}(70 \%)\end{array}$ & 0,124 & $3 \div 10$ & 6,5 & 7,8 \\
\hline
\end{tabular}

However, when using the samples №1 and №2 sweat fatty prints are faded-gray, which makes them visually indistinct on the trace (print) perceptive surface, some papillary lines have poor resolution. The prepared simulated powder consisting of the mixture of metallic iron and the pigment from iron-containing sludge (sample №4) has rich and bright colour. The intensity and image definition of the prints increases and papillary lines are well and clearly visible. This result can be achieved when the quantity of impurity clay materials in the pigment of iron containing sludge is up to $30 \%$. It is well-known that the color of iron oxide is getting lighter and becomes pure red-brown tint in the presence of about $5 \%$ of aluminum oxide [3], which allows to improve the print contrast and make it visually distinguishable on the print (trace) carrying surface. It also allows to increase the degree of manifestation of common and particular features of papillary lines structure.

\section{Conclusions}

"Developing" properties of ferromagnetic fingerprint powders are defined by the chemical nature and particle sizes of the pigment which compose the acidity of its surface, adhesive and magnetic properties, and color spectrum. The optimum ratio of these physical and chemical parameters is observed in the pigment consisting of iron-containing sludge tempered at $800^{\circ} \mathrm{C}$ which composition includes $\alpha-\mathrm{Fe}_{2} \mathrm{O}_{3}$ and impurity of clay materials.

\section{References}

1. State Standard -8135-74 Miniumiron. Specifications. Introduction 01.01.1974 with changes. - M.: Publishing Standards D999.

2. O.D. Lukashevich, N.T. Usova, V.A. Kutugin, V.A. Lotov The utilization of secondary products of water treatment in the production of iron oxide pigment for building materials //Water: Technology and Ecology. Problems and solutions. - 2011. - №2. - P.30-38

3. E.F. Belenky, I.V. Riskin. Chemistry and Technology of the pigments. Edition 4, revised and enlarged. - St. Petersburg: Chemistry, 1974. - 656 p. 